\title{
Blockage of the pyrimidine biosynthetic pathway affects riboflavin production in Ashbya gossypii
}

\author{
Rui Silva ${ }^{1}$, Tatiana Q. Aguiar ${ }^{1}$, Lucília Domingues* \\ CEB - Centre of Biological Engineering, University of Minho, 4710-057 Braga, Portugal
}

\section{A R T I C L E I N F O}

\section{Article history:}

Received 10 September 2014

Received in revised form 6 November 2014

Accepted 7 November 2014

Available online 15 November 2014

\section{Keywords:}

Ashbya gossypii

Pyrimidine pathway

Riboflavin production

Uridine/uracil auxotrophy

Nutritional stress

\begin{abstract}
A B S T R A C T
The Ashbya gossypii riboflavin biosynthetic pathway and its connection with the purine pathway have been well studied. However, the outcome of genetic alterations in the pyrimidine pathway on riboflavin production by $A$. gossypii had not yet been assessed. Here, we report that the blockage of the de novo pyrimidine biosynthetic pathway in the recently generated A. gossypii Agura3 uridine/uracil auxotrophic strain led to improved riboflavin production on standard agar-solidified complex medium. When extra uridine/uracil was supplied, the production of riboflavin by this auxotroph was repressed. High concentrations of uracil hampered this (and the parent) strain growth, whereas excess uridine favored the $A$. gossypii Agura3 growth. Considering that the riboflavin and the pyrimidine pathways share the same precursors and that riboflavin overproduction may be triggered by nutritional stress, we suggest that overproduction of riboflavin by the A. gossypii Agura3 may occur as an outcome of a nutritional stress response and/or of an increased availability in precursors for riboflavin biosynthesis, due to their reduced consumption by the pyrimidine pathway.
\end{abstract}

(c) 2014 Elsevier B.V. All rights reserved.

\section{Introduction}

The filamentous hemiascomycete Ashbya gossypii (syn. Eremothecium gossypii) is a remarkable riboflavin (vitamin B2) overproducer that has long been exploited for the industrial production of this vitamin (Stahmann et al., 2000; Kato and Park, 2012).

Riboflavin is synthesized from ribulose 5-phosphate (ribulose5P) and/or guanosine-5-triphosphate (GTP) through a pathway controlled by six genes (Schlösser et al., 2007). Riboflavin overproduction starts when the $A$. gossypii growth rate declines and is characterized by a strong increase in the expression of three of these genes ( $A g R I B 3, A g R I B 4$ and $A g R I B 5)$, which are regulated at the transcription level (Schlösser et al., 2001, 2007; Walther and Wendland, 2012). Environmental stress, such as nutritional (Schlösser et al., 2007) and oxidative stress (Kavitha and Chandra, 2009; Walther and Wendland, 2012), can trigger the overexpression of these genes and lead to riboflavin overproduction.

Since the riboflavin biosynthetic pathway shares its precursors with other metabolic pathways (Fig. 1), riboflavin production

\footnotetext{
* Corresponding author. Tel.: +351253 604400; fax: +351253604429.

E-mail address: luciliad@deb.uminho.pt (L. Domingues).

1 These authors contributed equally to this work.
}

is also influenced by alterations in these pathways (Park et al., 2011). Increased purine/GTP biosynthesis, either through the overexpression of genes encoding transcription factors (AgBAS1; Mateos et al., 2006) or enzymes directly involved in this pathway (AgPRS2-4, AgPRS3 or AgADE4) (Jiménez et al., 2005, 2008), or by increasing the intracellular availability of glycine (Monschau et al., 1998; Schlüpen et al., 2003; Kato and Park, 2006; Mateos et al., 2006), an important precursor for the de novo purine biosynthesis, leads in $A$. gossypii to increased riboflavin production. Alterations in the pyrimidine pathway are also expected to affect riboflavin production, as high concentrations of uracil in the medium inhibit riboflavin production by Eremothecium ashbyi (Goodwin and Pendlington, 1954). However, to our knowledge, the effect of manipulations in this pathway on riboflavin production by A. gossypii has not yet been addressed.

In the scope of the development of Cre-loxp-based tools for genetic manipulation of $A$. gossypii, we recently deleted the entire coding region of the A. gossypii ATCC 10895 AgURA3 gene with a dominant marker cassette that was subsequently removed using the Cre-recombinase, thus generating an A. gossypii uridine/uracil auxotrophic strain free of exogenous marker genes (Aguiar et al., 2014). In this study, we explored the growth and flavinogenic phenotypes of this strain (A. gossypii Agura3), which has the de novo pyrimidine biosynthetic pathway blocked at the orotidine5'-phosphate decarboxylase (AgUra3p) level (Fig. 1). 


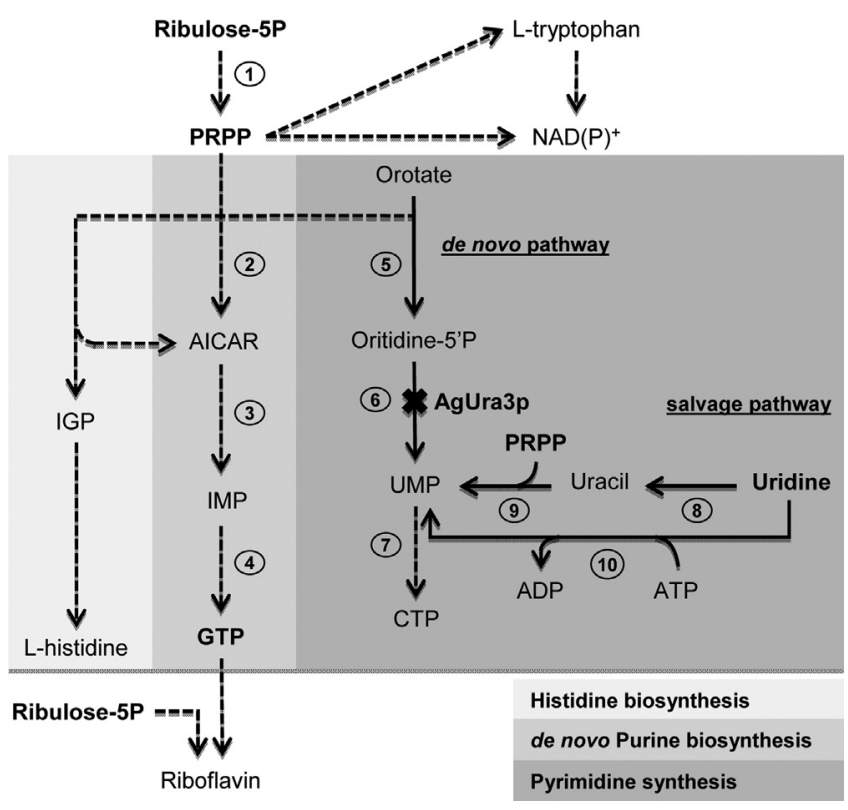

Fig. 1. Schematic representation indicating the contribution of ribulose-5P, PRPP and GTP to riboflavin biosynthesis and to other metabolic pathways. The $\times$ marks where the pyrimidine biosynthetic pathway is blocked in the A. gossypii Agura3 uridine/uracil auxotrophic strain. Dashed arrows indicate a multi-step pathway. IGP: D-erythro-imidazole-glycerol-phosphate; AICAR: 5amino-4-imidazolecarboxamide ribotide; IMP: 5'-inosine monophosphate; NAD(P): nicotinamide adenine dinucleotide (phosphate); CTP: cytidine-5'-triphosphate; ADP: adenosine-5'-pyrophosphate; ATP: adenosine-5'-triphosphate. Numbers indicate the enzymes involved in each step of the purine and pyrimidine pathways. 1: ribose-5-phosphate ketol-isomerase and PRPP synthetase; 2: PRPP amidotransferase, phosphoribosylglycinamidine synthetase, glycinamide ribotide transformylase, 5'-phosphoribosylformyl glycinamidine synthetase, phopshoribosylaminoimidazole synthetase, phosphoribosylamino-imidazole carboxylase, phosphoribosyl amino imidazolesuccinocarbozamide synthetase and adenylosuccinate lyase; 3: AICAR transformylase and IMP cyclohydrolase; 4: IMP dehydrogenase, guanosine-monophosphate synthase, guanylate kinase and nucleoside diphosphate kinase; 5: orotate phosphoribosyltransferase; 6: orotidine-5'-phosphate decarboxylase; 7: uridylate kinase and CTP synthase; 8: uridine nucleosidase; 9: uracil phosphoribosyltransferase; 10: uridine kinase.

\section{Materials and methods}

Spores from A. gossypii ATCC 10895 (provided by Prof. P. Philippsen, Basel University) and from its uridine/uracil auxotrophic derivate Agura3 (Agura3 $\Delta::$ loxP; Aguiar et al., 2014) were prepared and stored as previously described (Aguiar et al., 2014).

Agar-solidified Ashbya Full Medium (AFM; Altmann-Jöhl and Philippsen, 1996) or Synthetic Complete (SC) medium buffered with $1 \mathrm{~g} / \mathrm{LCaCO}_{3}$ and containing $20 \mathrm{~g} / \mathrm{L}$ glucose (Ribeiro et al., 2012) were inoculated with $10^{5}$ spores and incubated at $30^{\circ} \mathrm{C}$. When indicated, AFM and SC were supplemented with uridine/uracil.

Colony radial growth was determined by measuring the diameter of colonies grown on agar-solidified medium in $90 \mathrm{~mm}$ diameter Petri dishes. For total riboflavin estimation, the mycelium from each plate was collected after 3 days of growth and digested with $4 \mathrm{mg} / \mathrm{mL}$ Lysing Enzymes from Trichoderma harzianum (Sigma-Aldrich) for $1 \mathrm{~h}$. Cell debris was removed by centrifugation and the supernatant's absorbance was measured at $445 \mathrm{~nm}$, and converted into riboflavin concentration using a standard curve constructed with pure riboflavin standards (Sigma-Aldrich). Cell dry weight was measured after drying the collected mycelium at $105^{\circ} \mathrm{C}$ and specific riboflavin production was calculated as $\mathrm{mg}_{\text {riboflavin }} / \mathrm{g}_{\text {mycelium}}$.

\section{Results and discussion}

The A. gossypii Agura3 auxotrophic strain requires the supply of exogenous uridine/uracil to compensate its nutritional deficiency (Fig. 2A) (Aguiar et al., 2014). However, in this work uracil was shown to be detrimental for the A. gossypii growth (Fig. $2 \mathrm{~A}$ and $B)$. In AFM, the growth inhibitory effect of high concentrations of uracil was more evident in the Agura3 strain than in its parent strain (Fig. 2B). Nevertheless, in SC medium, uracil clearly showed to negatively affect the parent strain growth as well (Fig. 2A). Similar concentrations of uracil were reported not to affect the growth of the A. gossypii close relative E. ashbyi (Goodwin and Pendlington, 1954). By contrast, uridine did not cause growth inhibition (Fig. 2A and B). On the contrary, it significantly favored the Agura3 strain growth, which only reached the level of the parent strain growth under extra uridine supplementation conditions (Fig. 3).

Excess of exogenous uracil (but not uridine) was recently reported to have a strong inhibitory effect on the Aspergillus nidulans growth, being an auxotrophic strain deleted in pyrG (encoding orotidine-5'-phosphate decarboxylase) also more sensitive to uracil than the wild type strain (Sun et al., 2013). This uracil toxicity is believed to be related with the misincorporation of uracil into DNA when uracil is accumulated in excess (Sun et al., 2013). Uridine cannot be directly incorporated into DNA, which may explain why excess uridine did not cause growth inhibition in A. nidulans (Sun et al., 2013) and A. gossypii.

The A. gossypii Agura3 strain presented a riboflavin overproducing phenotype on standard agar-solidified AFM, detected by the yellow color of the colonies, which was not displayed by the parent strain under the same conditions (Fig. 2C). However, when increasing concentrations of uridine/uracil were added to AFM, the production of riboflavin by A. gossypii Agura3 gradually diminished (Fig. 2D and E), whereas the flavinogenic phenotype of the parent strain remained unaffected ( $c f$. Fig. 2B-D). Addition of $0.5 \mathrm{mM}$ uridine to AFM resulted in an approximately ninefold decrease in riboflavin production by A. gossypii Agura3 and, as mentioned above, in improved growth (Fig. 3). High concentrations of uracil in AFM also inhibited riboflavin production by A. gossypii Agura3, but lead instead to decreased growth (Fig. 2B and $\mathrm{E}$ ). Hence, the riboflavin overproducing phenotype of this uridine/uracil auxotroph is directly correlated with the accessibility to these nutrients.

Nutritional stress can trigger riboflavin overproduction in $A$. gossypii (Schlösser et al., 2007). The cascade of events involved in this stress response has not been fully elucidated, but it is known to induce the expression of genes of the riboflavin biosynthetic pathway, thus increasing the metabolic flux to riboflavin synthesis (Schlösser et al., 2007; Walther and Wendland, 2012).

The metabolic flux to riboflavin synthesis is also determined by the amount of precursors available (Kato and Park, 2012). Ribulose$5 \mathrm{P}$ is obtained from the carbohydrate metabolism through the oxidative branch of the pentose phosphate pathway and can be directly used as a precursor for riboflavin synthesis or isomerized to ribose-5-phosphate (Park et al., 2011). Ribose-5-phosphate is in its turn required for the synthesis of phosphoribosyl pyrophosphate (PRPP), an intermediate metabolite necessary for the synthesis of other riboflavin precursor (GTP), purine, pyrimidine and pyridine nucleotides, histidine and tryptophan (Fig. 1) (Jiménez et al., 2008). Increased PRPP synthetase activity led in A. gossypii to increased riboflavin production (Jiménez et al., 2008). Moreover, a riboflavin overproducing $A$. gossypii mutant isolated by disparity mutagenesis displayed threefold higher expression of PRPP synthetase encoding genes during the riboflavin production phase than its parent strain (Park et al., 2011). Therefore, alterations in the intracellular PRPP pool affect riboflavin production in A. gossypii. 
A
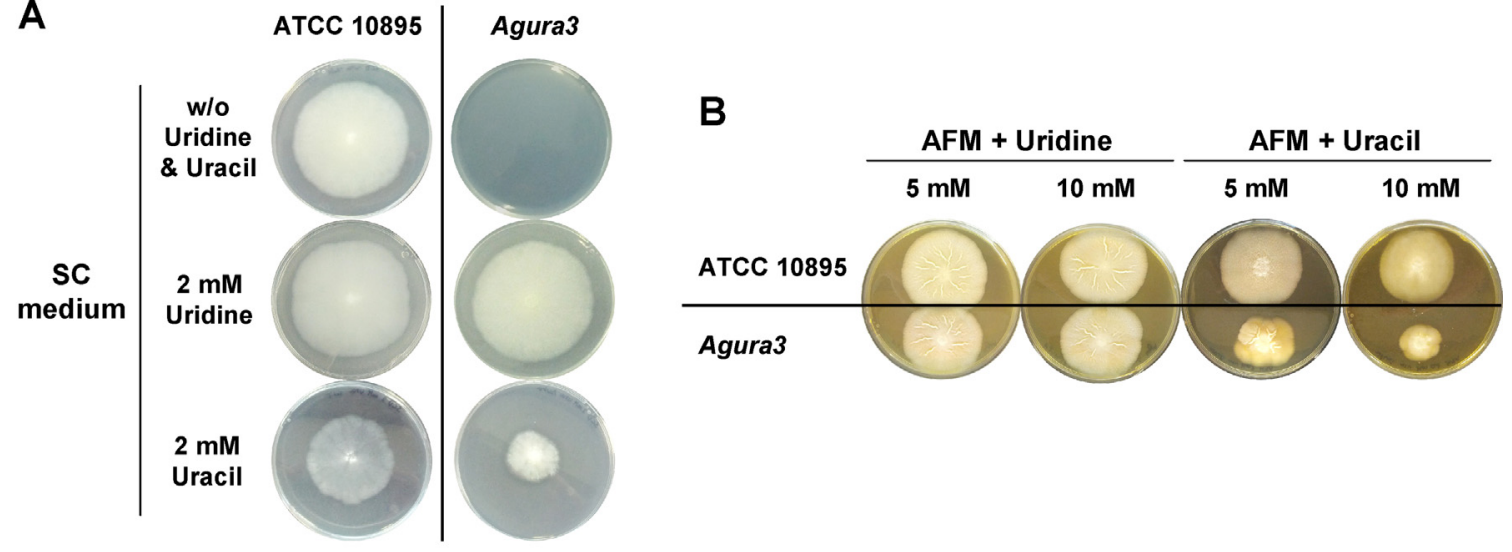

C ATCC $10895 \quad$ Agura3

AFM

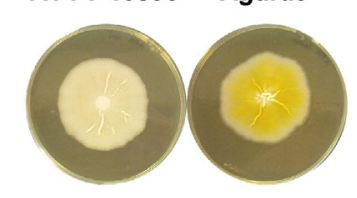

D
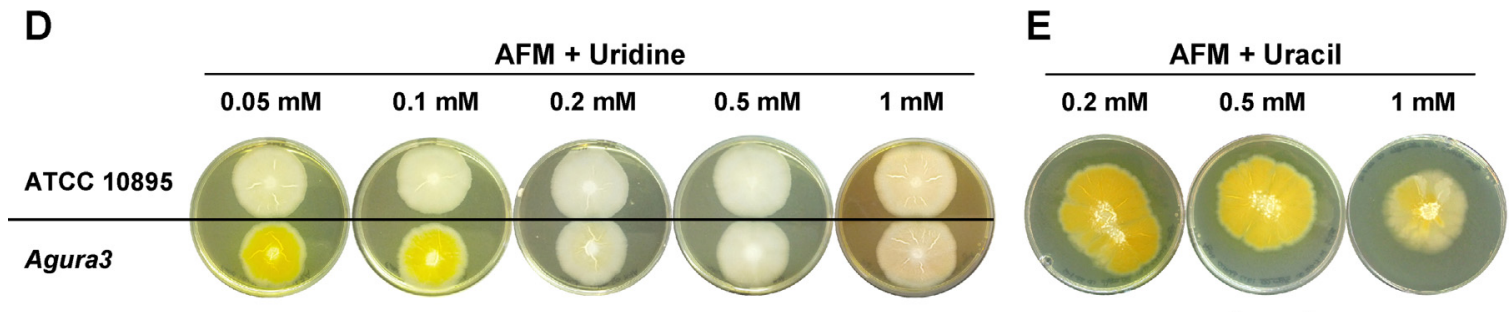

Agura3

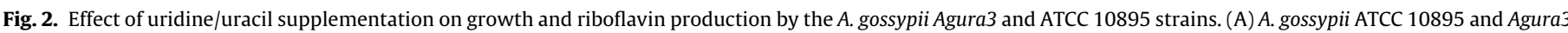

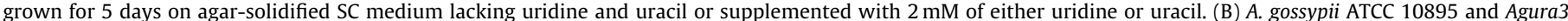

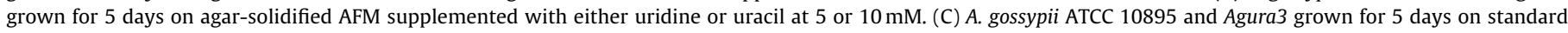

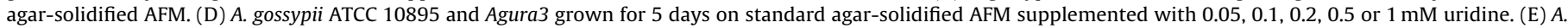
gossypii Agura3 grown for 8 days on agar-solidified AFM containing 0.2, 0.5 or $1 \mathrm{mM}$ uracil. All images are representative of three biological replicas.

\begin{tabular}{lcc}
\hline & AFM & $\begin{array}{c}\text { AFM + 0.5 mM } \\
\text { Uridine }\end{array}$ \\
\hline A. gossypii ATCC 10895 & & \\
\hline Riboflavin (mg/gmycelium) & $1.0 \pm 0.2^{\mathrm{a}}$ & $0.9 \pm 0.2^{\mathrm{a}}$ \\
Colony radial growth (mm) & $27.4 \pm 0.4^{\mathrm{b}}$ & $27.3 \pm 0.3^{\mathrm{b}}$ \\
\hline A. gossypii Agura3 & & \\
\hline Riboflavin (mg/gmycelium) & $7.5 \pm 0.4^{\mathrm{c}}$ & $0.8 \pm 0.2^{\mathrm{a}}$ \\
Colony radial growth (mm) & $25.3 \pm 0.3^{\mathrm{d}}$ & $26.8 \pm 0.1^{\mathrm{b}}$ \\
\hline
\end{tabular}

$\mathrm{a}, \mathrm{c}-p<0.0001 ; \mathrm{b}, \mathrm{d}-p<0.0001$

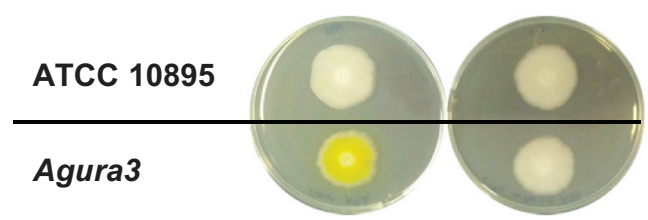

Fig. 3. Colony radial growth and specific riboflavin production by A. gossypii ATCC 10895 and Agura3 after 3 days of growth on standard agar-solidified AFM and AFM supplemented with $0.5 \mathrm{mM}$ uridine. The values represent the mean \pm standard deviation of three biological replicas. One-way ANOVA and Tukey's test were used to find means significantly different from each other. The images are representative of the indicated values.
Given that the purine and pyrimidine synthesis was estimated to consume most of the synthesized PRPP (Hove-Jensen, 1988) and that A. gossypii Agura3 has the de novo pyrimidine biosynthetic pathway blocked (Fig. 1), an increase in the PRPP available for riboflavin synthesis through the de novo purine/GTP biosynthetic pathway and/or a reduction in PRPP synthesis driven by its intracellular accumulation, with consequent increased availability of ribulose-5P for riboflavin synthesis, might have occurred when no extra uridine/uracil was supplied. Any of these events could explain the overproduction of riboflavin by this strain under these conditions. Moreover, increased flux in the riboflavin pathway triggered by a nutritional stress response may have also contributed to this phenotype. Conversely, when extra uridine/uracil was supplied, the nutritional requirements of this auxotrophic strain were gradually compensated, thus preventing nutritional stress. Additionally, the synthesis of uridine monophosphate (UMP) from uridine/uracil (Fig. 1) must have increased, therefore reducing the PRPP availability for GTP/riboflavin synthesis. Increased demand for PRPP may have also led to the redirection of ribulose-5P for its synthesis.

Our findings show that blockage of the A. gossypii pyrimidine biosynthetic pathway led to increased riboflavin production, which was correlated with the accessibility to the nutrients required to compensate the auxotrophy caused by this blockage. Although changes in the purine pathway have been described to affect riboflavin production, these are the first experimental results documenting alterations in riboflavin production caused by metabolic engineering of the pyrimidine pathway. 


\section{Acknowledgments}

This work was supported by Fundação para a Ciência e a Tecnologia, Portugal, through Project AshByofactory PTDC/EBBEBI/101985/2008 - FCOMP-01-0124-FEDER-009701, Project GlycoCBMs FCTPTDC/AGR-FOR/3090/2012 - FCOMP-01-0124-FEDER027948, Strategic Project PEst-OE/EQB/LA0023/2013, and Project BioInd NORTE-07-0124-FEDER-000028 co-funded by the Programa Operacional Regional do Norte (ON.2 - O Novo Norte), QREN, FEDER. The technical support provided by Cláudia Dinis is also acknowledged.

\section{References}

Aguiar, T.Q., Dinis, C., Domingues, L., 2014. Cre-loxP-based system for removal and reuse of selection markers in Ashbya gossypii targeted engineering. Fungal Genet. Biol. 68, 1-8.

Altmann-Jöhl, R., Philippsen, P., 1996. AgTHR4, a new selection marker for transformation of the filamentous fungus Ashbya gossypii, maps in a four-gene cluster that is conserved between A. gossypii and Saccharomyces cerevisiae. Mol. Gen. Genet. 250, 69-80.

Goodwin, T.W., Pendlington, S., 1954. Studies on the biosynthesis of riboflavin, Nitrogen metabolism and flavinogenesis in Eremothecium ashbyii. Biochem. J. 57, 631-641.

Hove-Jensen, B., 1988. Mutation in the phosphoribosylpyrophosphate synthetase gene (prs) that results in simultaneous requirements for purine and pyrimidine nucleosides, nicotinamide nucleotide, histidine, and tryptophan in Escherichia coli. J. Bacteriol. 170, 1148-1152.

Jiménez, A., Santos, M.A., Pompejus, M., Revuelta, J.L., 2005. Metabolic engineering of the purine pathway for riboflavin production in Ashbya gossypii. Appl. Environ. Microbiol. 71, 5743-5751.

Jiménez, A., Santos, M.A., Revuelta, J.L., 2008. Phosphoribosyl pyrophosphate synthetase activity affects growth and riboflavin production in Ashbya gossypii. BMC Biotechnol. 8, 67-78.
Kato, T., Park, E.Y., 2006. Expression of alanine: glyoxylate aminotransferase gene from Saccharomyces cerevisiae in Ashbya gossypii. Appl. Microbiol. Biotechnol. 71, 46-52.

Kato, T., Park, E.Y., 2012. Riboflavin production by Ashbya gossypii. Biotechnol. Lett. 34, 611-618.

Kavitha, S., Chandra, T.S., 2009. Effect of vitamin E and menadione supplementation on riboflavin production and stress parameters in Ashbya gossypii. Proc. Biochem. 44, 934-938.

Mateos, L., Jiménez, A., Revuelta, J.L., Santos, M.A., 2006. Purine biosynthesis riboflavin production, and trophic phase span are controlled by a Myb-related transcription factor in the fungus Ashbya gossypii. Appl. Environ. Microbiol. 72, 5052-5060.

Monschau, N., Sahm, H., Stahmann, K.P., 1998. Threonine aldolase overexpression plus threonine supplementation enhanced riboflavin production in Ashbya gossypii. Appl. Environ. Microbiol. 64, 4283-4290.

Park, E.Y., Ito, Y., Nariyama, M., Sugimoto, T., Lies, D., Kato, T., 2011. The improvement of riboflavin production in Ashbya gossypii via disparity mutagenesis and DNA microarray analysis. Appl. Microbiol. Biotechnol. 91, 1315-1326.

Ribeiro, O., Domingues, L., Penttilä, M., Wiebe, M.G., 2012. Nutritional requirements and strain heterogeneity in Ashbya gossypii. J. Basic Microbiol. 52, 582-589.

Schlösser, T., Schmidt, G., Stahmann, K.P., 2001. Transcriptional regulation of 3,4dihydroxy-2-butanone 4-phosphate synthase. Microbiology 147, 3377-3386.

Schlösser, T., Wiesenburg, A., Gätgens, C., Funke, A., Viets, U., Vijayalakshmi, S., Nieland, S., Stahmann, K.P., 2007. Growth stress triggers riboflavin overproduction in Ashbya gossypii. Appl. Microbiol. Biotechnol. 76, 569-578.

Schlüpen, C., Santos, M.A., Weber, U., De Graaf, A., Revuelta, J.L., Stahmann, K.P. 2003. Disruption of the SHM2 gene, encoding one of two serine hydroxymethyltransferase isozymes, reduces the flux from glycine to serine in Ashbya gossypii. Biochem. J. 369, 263-273.

Stahmann, K.P., Revuelta, J.L., Seulberger, H., 2000. Three biotechnical processes using Ashbya gossypii, Candida famata, or Bacillus subtilis compete with chemical riboflavin production. Appl. Microbiol. Biotechnol. 53, 509-516.

Sun, X.Y., Zhu, J.F., Bao, L., Hu, C.C., Jin, C., Harris, S.D., Liu, H.W., Li, S.J., 2013. pyrC is required for maintaining stable cellular uracil level and normal sporulation pattern under excess uracil stress in Aspergillus nidulans. Sci. China Life Sci. 56, 467-475.

Walther, A., Wendland, J., 2012. Yap1-dependent oxidative stress response provides a link to riboflavin production in Ashbya gossypii. Fungal Genet. Biol. 49, 697-707. 